\title{
Molecular Chirality as an Integral Biomarker of Lactation, Nutrition and Cognitive Development Express-Review
}

\author{
Victor V Dyakin ${ }^{1 *}$ and Eva V Posner ${ }^{2}$ \\ ${ }^{1}$ Head of lab Perception of Virtual Reality La, The Nathan S Kline Institute for Psychiatric Research (NKI), USA \\ ${ }^{2}$ Phelps Hospital, Northwell Health, The Baby-Friendly Hospital Initiative (BFHI), USA \\ *Corresponding author: Victor V Dyakin, Head of lab Perception of Virtual Reality La, The Nathan S Kline Institute for Psychiatric \\ Research (NKI), USA. \\ To Cite This Article: Victor V Dyakin, Eva V Posner, Abel Lajtha. Molecular Chirality as an Integral Biomarker of Lactation, Nutrition, and \\ Cognitive Development Lactation Express. 2020 - 8(6). AJBSR.MS.ID.001337. DOI: 10.34297/AJBSR.2020.08.001337.
}

Received: 鮆April 23, 2020; Published: 眥 May 22, 2020

\begin{abstract}
It becomes an axiom that all living organisms are composed predominantly of L-amino acids (L-AAs) and D-sugars. However, in many biomedical studies, there is a significant discrepancy between the amount of available information regarding D-AAs biology and a degree of pragmatic implementation. It is applying to the fields of nutrition and lactation. The mutual interaction of internal and environmental sources of D-AAs in an organism, including the food, microorganisms, is influenced by the spontaneous and enzymatic racemization. The interacting sources of D-AAs are recognized as an essential determinant in the embryonic/infant development, the lifestyle of an adult an organism, and aging. Progress in the analytical methods of chiral discrimination is promising to revolutionize the field of neuroscience, neurodegeneration, psychology, drug production, and the food industry, which should be reflected in the field of lactation. However, the attention to the D/L AAs balance in the area of breastfeeding is overlooked until the present. In short, our aim is predicting the importance of the serine racemase (SerRs) enzymes in human breast milk production and digestion.
\end{abstract}

Keywords: Lactation; Enzyme; Serine Racemase; Racemization; Post-Translational Modification; Protein Folding; Biochirality

Abbreviations: L/D-AAs: L/D-amino acids; SerR: Serine Racemase; DAAO: D-AA oxidase; D-Ala: D-Alanine; D-Asp: D-aspartic Acid; D-Arg: D-arginine; D-Glu: D-glutamic acid; D-Gln: D-glutamine; D-Leu: D-leucine; D-Lys: D-lysine; D-Met: D- Methionine; D-Ph: D-phenylalanine; D-Ser: D-serine; PTMs: Post-translational modifications; PhTs ${ }^{\mathrm{NE}}$ : Non-Equilibrium Phase Transitions; SerRs: Serine Racemase; DAO, DAAO: D-Amino Acid Oxidase

\section{Introduction}

\section{Biochirality}

"D-amino acids, the enantiomeric counterparts of L-amino acids, were long considered to be non-functional or not even present in living organisms" [1]. "D-Amino acids are now emerging as potential biomarkers" [2]. "Accumulating evidence points to distinctive roles of D-AAs in non-ribosomal physiology" [3]. "Chiral recognition is the principle governing most biomolecular interactions" [4]. Molecular biochirality chirality (BioCh)* has its physical and chemical ground [4,5]. Our concern is the molecular BioCh in relation to the science of nutrition. Under the impact of new coming results, the intuitive impression of the absolute biological homo-chirality is replaced by the concept of prevalent chirality. Consequently, the descriptive terminology is shifted from "mysterious","attractive," and "surprising" to the simple and objective "fundamental". It becomes an axiom that all living organisms are composed predominantly of L-amino acids (L-AAs) and D-sugars. The main features characterizing a new level of understanding are.

a) The physiology of an organism is driven by the fine dynamical balance between the prevalent and inferior (L- and D-) isoforms [6].

b) The primary sources of D-AAs in the organism are the enzymatic and spontaneous post-translational modifications (PTM). The significant part of D-AAs pool (from10- to $20 \%$ ) in different organs of an organism (such as kidney, brain, and others) is independent on the racemase enzymes activity $[7,8]$.

c) The physical basis of PTMs of protein folding is the nonequilibrium phase transitions $\left(\mathrm{PhTs}^{\mathrm{NE}}\right)$ [9]. 
The physical basic of the prevalent AAs chirality (as a deviation from an equilibrium racemic state (antiracemization), and the tendency to spontaneous racemization is grounded on the thermodynamics laws. The quantitative relation between the entropy and energy are reviling the Parity Violation Energy Difference (PVED) in the organic molecules [6].

d) An enormous number of macromolecular spatial transformations require the statistical interpretation of the thermodynamics of PTMs.

e) The transformation of protein's spatial symmetry is inevitably accompanied by the alterations of the corresponding thermodynamic state.

f) Spontaneous, nonenzymatic reactions in proteins (including racemization) are known to have the relevance to protein misfolding, aggregation, aging, neurodegeneration, and nutrition. Current evidence indicates that D-AAs are present in the high concentration in microorganisms and the lower level in plants, mammals and humans [7]. Such evolutionary consistency is associated with a variety of life-supporting cellular functions, including cell division, cell proliferation, and cell signaling. Involvement of D-AAs in neurotransmission underlies their role in the laterality of brain structure and functions [9,10]. These functions include learning, memory, spatial perception, and control of behavior. Currently, molecular chirality is widely recognized as a useful biomarker in the epidemiology and pharmacology [11-13]. At the same time, it is just at the very initial phase in the field of nutrition [14-16].

\section{Source of D-AAs in Human Organism}

\section{Post-Translational Modifications}

There are several internal and environmental sources of D-AAs in an organism which are influenced by the mutual interaction. Among them, the most frequently considered are the food, microorganisms, and different forms of racemization [1,17]. The primary internal mechanism underlying the appearance of D-AAs containing peptides/proteins is attributed to the enzymatic and spontaneous post-translational racemization (Figure 1). Biogenesis (synthesis and degradation) of D-AAs containing peptides/proteins is performed by the catholic activity of specific enzymes, including serine racemase (SerR) and D-AA oxidase (DAAO) [18]. According to many experimental evidence, D-Ser plays a distinct role in the biochirality of the food [19], the mammal's physiology and neurotransmission $[20,21]$. The reason for the specificity of D-Ser in the biochemistry of an organism remain to be studied.

In the organism, D-Ser is synthesized by SerR and degraded by DAAO 20. D-Ser level in mammals is highest in urine and serum [22]. The key regulator of intra-body chiral AAs level (including D-Ser) is urinary excretion by the kidney [23]. PTMs include both the intrinsic spontaneous and enzyme-driven racemization [1]. As the consequence, D-AAs are detected in all major areas, including kidney, liver, skin and the brain [23-28].

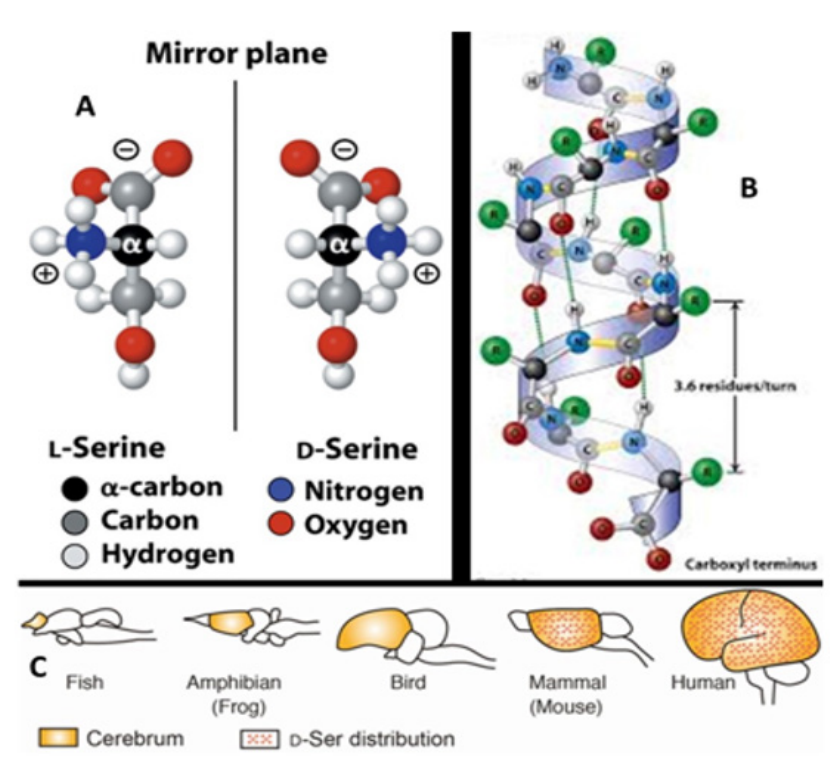

Figure 1: $A, B, C$

A. All living organisms are composed predominantly of L-amino acids (L-AAs). Example of the Serine (Ser) amino acid.

B. The $L$ and $D$-isoforms of $A A s$ are different in the way the chain of $A A s$ is folding into the secondary and higher-order structures a result of the angle of the atomic bond. Example of alpha-helix. Adopted from \{https://socratic.org/questions/differences-between-alpha-helix-and-betapleated-sheet-structures-of-pro).

C. Distribution of D-Ser in the CNS of vertebrates. Typical vertebrate brain structures are shown with the cerebrum highlighted in ocher. D-Ser (red dots) occurs exclusively in mammalian (including human) cerebrum. Adopted from \{Adopted from [53-54]. 


\section{Microbiota}

The interactions between epithelial cells and luminal microbiota have a significant impact on host nutrition and health through the metabolism of dietary components [29]. Microbiota is a critical player in the age-dependent (from infant to adult) food consumption. Notable, that in mammals, many D-AAs are produced by microbiota (gut and intestinal), including D-Ala, D-Arg, D-Asp, D-Gln, D- Glu, D-Leu, D-Lys, D-Met, D-Phe, D-Ser, and D-Trp [30]. Many of them are known as racemization prone. According to recent evidence, about $30 \%$ of the human D-AAs are derived from microbial synthesis [31-33]. Microbial D-AAs are involved in communication with the host D-AAs [3]. The essential role of D-AAs recognition in the host-mircobe interface is supported by many experimental facts including following that "D-phenylalanine and D-tryptophan regulate chemotaxis of neutrophils through a G- coupled protein receptors, D-Ser has a bacteriostatic role in the urinary tract, $\mathrm{D}-\mathrm{Ph}$, and D-Leu inhibit innate immunity through the sweet taste receptor in the upper airway, and D-Trp modulates immune tolerance in the lower airway" [32]. In this situation, it is not surprising that age-related alteration in the intestinal microbiota found to be associated with the brain amyloid-beta (A-beta) plaque deposition [34]. The molecular mechanism of such association could be the microbiota-induced expression of the enzymes (such as D-AAs racemases** and DAO). **Currently, two racemase enzymes have been found in mammals signaling pathways: Ser-Rs and Asp-Rs 17. Of these two enzymes, only SerRs can be found in human tissue [1]. D-Ser and D-Asp are (so far) the only D-AAs in humans originating from intrinsic racemization [1]. Both enzymes are previously unrecognized mediators of microbehost interplay, including microbiota-induced racemization.

\section{Diet: Food Processing and Racemization}

The presence of D-AAs in foods, including milk, beer, wine [35-38], vegetables, fruit and rice [19,38] is well known. Over the past decades, it has become clear that food processing techniques contribute to the racemization of L-AAs into D-AAs [1]. However, the studies devoted to the role of internal racemization in the digestion are practically absent.

\section{Conclusion}

Progress in the analytical methods of chiral discrimination is promising to revolutionize the field of neuroscience, neurodegeneration, psychology, drug production, food industry and lactation. A relevant example is biomarkers in kidney pathology, which, until recently, was a lock of effective biomarkers to the predictive prognosis of chronic kidney disease. The attention to peptide/protein chirality opens a new era of diagnostic and treatment [23]. However, in many biomedical studies, there is a significant discrepancy between the amount of available information regarding D-AAs biology and a degree of implementation. It is applying to the field of lactation, due to the fact, that D-AAs are the essential players in the embryonic and infant development [40, 41]. Milk proteins of different animals have a high and wide range of the AAs concentrations, which are the subjects of racemization. Consequently, the significance of breastfeeding should be reconsidered from the prism of biochirality. Indeed about 20 necessary and not essential AAs found in the breast milk and AAs estimation is a routine element of human milk protein quality control. It is well known that the heating of natural and artificial milk is accompanied by racemization [42]. It is also widely accepted that gut microbiota is an essential mediator of breast milk) impact on the host body AAs metabolism $[43,44]$. However, attention to the D/L AAs balance is overlooked until the present.

The rare exception is the several recently published review $[1,45]$ which can be recommended as a useful stereo-chemistryrelated introduction to nutritionists. Currently, only a little data exists on D-AAs needs in infants and children [46]. Therefore, the role of D-AAs in this field is only on the way to appreciate it. The new strategy of breastfeeding is focused on education to convince that both of two factors are essential: the most effective role of the immediate skin-to-skin contact of a mother with an infant, and the specificity of D-AA's contents. Nonetheless, the attention to the degree of AAs racemization remains to be low or absent. It is known that milk proteins are "predigested" by the diverse family proteolytic enzymes within the human mammary gland $[47,48]$. The importance of enzymes in human breast milk production and digestion by infants was both predicted and demonstrated [4,47-49]. These experimental facts are in sharp contrast with the situation regarding the enzymes of the post-translational racemization role in the breastfeeding. The implication of AAs racemases in the preparation of milk products is known for the centuries [50]. However, currently an exploration of the enzymes of human milk does not extend to the action of the racemase enzymes (widely expressed in an organism). Based in this discrepancy we are predicting the importance of the serine racemase (SerRs) enzymes family in human breast milk production and digestion. It is notable, that L-Serine (L-Ser) \{non-essential amino acid\}, is required to synthesize membrane lipids such as phosphatidylserine and sphingolipids [51]. Ser-L deficiency inevitably compromises cell membrane function [52-54] Esaki et al. Therefore, excessive racemization can be also harmful to all significant cellular functions, including the processes of milk production in an organism of mother and milk digestion by infants. Intake of proteins is the key nutritional factors in human diet. The balance of plant and animal proteins has a differential significance for aging stage from infant to ageing adults. At the early stage the developmental aspects are the primary concerns. From the third decade of life a gradual decline in muscle mass and alteration of muscle morphology gain the significance $[55,56]$. These results have an immediate link to the fundamental role of biochirality in general, prevalence of 
L-amino acids, and protein aging in human physiology. Giving the fact that abnormal D-AAs level is associated with the chronic and acute kidney diseases [57] and recent spark of interest to link the brain-gut-microbiome axis, [58, 59] it is logical to assume that the nutritional aspects of protein chirality are the essential determinants in the complex of issues: from the early development to the age-related decline of the perceptual and cognitive function [59].

\section{References}

1. Bastings JJAJ, Van Eijk HM, Damink SWO, Rensen S (2019) D-amino Acids in Health and Disease: A Focus on Cancer. Nutrients 11(9): 2205.

2. Sasabe J, Suzuki M (2018) Emerging Role of d-Amino Acid Metabolism in the Innate Defense. Front Microbiol 9: 933.

3. Baykusheva D, Zindel D, Svoboda V, Bommeli E, Ochsner M, et al. (2019) Real-time probing of chirality during a chemical reaction. PNAS 116(48): 23923-23929.

4. Fujiki M (2014) Supramolecular Chirality: Solvent Chirality Transfer in Molecular Chemistry and Polymer Chemistry. Symmetry 6(3): 677-703.

5. Santos ACL, Muniz CR, Oliveira LT, Souza JT (2019) Contributions of the 4-D Chern-Simons Electrodynamics to the Molecular Biochirality. Physics.

6. Genchi G (2017) An overview on D-amino acids. Amino Acids 49(9): 1521-1533.

7. Horio M, Kohno M, Fujita Y, Ishima T, Inoue R, et al. (2011) Levels of $\mathrm{D}$-serine in the brain and peripheral organs of serine racemase (Srr) knock-out mice. Neurochem Int 59(6): 853-859.

8. Dyakin VV, Lucas J (2017) Non-equilibrium Phase Transition in Biochemical-Systems. Chain of Chirality Transfer as Determinant of Brain Functional Laterality. Relevance to Alzheimer Disease and Cognitive Psychology. The J of the Alzheimer's Association 13(7): 1498.

9. Dyakin VV, Lucas J, Dyakina Fagnano NV, Posner EV, Vadasz C (2017) Chain of Chirality Transfer as Determinant of Brain Functional Laterality. Breaking the Chirality Silence: Search for New Generation of Biomarkers. Relevance to Neurodegenerative Diseases, Cognitive Psychology and Nutrition Science. NNR Neurology and Neuroscience Research 1(1): 2.

10. Castrignanò E, Mardal M, Rydevik A, Miserez B, Ramsey J, et al. (2018) A new approach towards biomarker selection in estimation of human exposure to chiral chemicals: a case study of mephedrone. Scientific Reports 8(1): 4295.

11. Evans SE, Davies P, Lubben A, Kasprzyk Hordern B (2015) Determination of chiral pharmaceuticals and illicit drugs in wastewater and sludge using microwave assisted extraction, solid-phase extraction and chiral liquid chromatography coupled with tandem mass spectrometry. Anal Chim Acta 2(882): 112-126.

12. Sanganyado E, Lu Z, Fu Q Schlenk D, Gan J (2017) Chiral pharmaceuticals: A review on their environmental occurrence and fate processes. Water Res 1(124): 527-542.

13. Lorenzo MP, Dudzik D, Varas E, Gibellini M, Skotnicki M, et al. (2015) Optimisation and validation of chiral GM-MS method for determination of free D-amino acid ration in human urine: application to a gestational diabeties mellitus study. J Pharm Biomed Anal 25(107): 480-487.

14. Stipanuk MH, Caudill MA (2016) Biochemical, Physiological and Molecular Aspects of Human Nutrition. Elsvier p.968.

15. He Tian, Zheng N, Li S, Zhang Y, Zhao S, et al. (2017) Characterization of chiral amino acids from different milk origins using ultra-performance liquid chromatography coupled to ion-mobility mass spectrometry. Sci Rep 10(7): 46289.
16. Ohide H, Miyoshi Y, Maruyama R, Hamase K, Konno R (2011) D-Amino acid metabolism in mammals: biosynthesis, degradation and analytical aspects of the metabolic study. J Chromatogr B Analyt Technol Biomed Life Sci 879(29): 3162-3168.

17. Graham DL, Beio ML, Nelson DL, and Berkowitz DB (2019) Human Serine Racemase: Key Residues/Active Site Motifs and Their Relation to Enzyme Function. Frontiers in Molecular Neuroscience 6: 8.

18. Gogami Y, Ito K, Kamitani Y, Matsushima Y, Oikawa T (2009) Occurrence of D-serine in rice and characterization of rice serine racemase. Phytochemistry 73(3): 380-387.

19. Ito T, Hamauchi N, Hagi T, Morohashi N, Hemmi H, et al. (2018) D-Serine Metabolism and Its Importance in Development of Dictyostelium discoideum. Front Microbiol 9: 784

20. Neame S, Safory H, Radzishevsky I, Touitou A, Marchesani F, Marchetti M, Kellner S, et al. (2019) The NMDA receptor activation by d-serine and glycine is controlled by an astrocytic Phgdh-dependent serine shuttle. PNAS 116(41): 20736-20742.

21. Brauer AL, White AN, Learman BS, Johnson AO, Armbruster CE (2019) D-Serine Degradation by Proteus mirabilis Contributes to Fitness during Single-Species and Polymicrobial Catheter-Associated Urinary Tract Infection. Am Soc for Microbiology Host-Microbe Biology. mSphere 4:1.

22. Hesaka A, Sakai S, Hamase K, Ikeda T, Matsui R, et al. (2019) D-Serine reflects kidney function and diseases Scientific Reports 5104.

23. Kimura T, Hamase K, Miyoshi Y, Yamamoto R, Yasuda K, et al. (2016) Chiral amino acid metabolomics for novel biomarker screening in the prognosis of chronic kidney disease. Sci Rep 6: 26137.

24. Hesaka A, Sakai S, Hamase K, Ikeda T, Matsui R, et al. (2019) D-Serine reflects kidney function and diseases. Scientific Reports 9(1): 5104.

25. Benneyworth MA, Li Y, Basu AC, Bolshakov VY, Coylec JT (2012) Cell Selective Conditional Null Mutations of Serine Racemase Demonstrate a Predominate Localization in Cortical Glutamatergic Neurons. Cell Mol Neurobiol 32(4): 613-624.

26. Fujii N (2005) D-amino acid in elderly tissues. Biol Pharm Bull 28(9): 1585-1589.

27. Kiriyama Y, Nochi H (2006) D-Amino Acids in the Nervous and Endocrine Systems. Scientifica 6494621.

28. Yang Z, Liao SF (2019) Physiological Effects of Dietary Amino Acids on Gut Health and Functions of Swine. Front Vet Sci 6: 169.

29. Matsumoto M, Kunisawa A, Hattori T, Kawana S, Kitada Y, et al. (2018) Free D-amino acids produced by commensal bacteria in the colonic lumen. Scientific Reports 8: 17915.

30. Friedman M (2010) Origin, Microbiology, Nutrition, and Pharmacology of D-Amino Acids. Chem Biodivers 7(6): 1491-1530.

31. Sasabe J, Miyoshi Y, Rakoff Nahoum S, Zhang T, Mita M, et al. (2016) Interplay between microbial d-amino acids and host d-amino acid oxidase modifies murine mucosal defence and gut microbiota. Nat Microbiol 1(10): 16125

32. Aliashkevich A, Alvarez L, Cava F (2018) New Insights into the Mechanisms and Biological Roles of d-Amino Acids in Complex EcoSystems. Front Microbiol 9: 683.

33. Cox LM, Schafer MJ, Sohn J (2019) Calorie restriction slows age-related microbiota changes in an Alzheimer's disease model in female mice. Sci Rep 9: 17904

34. Rubio Barroso S, Santos Delgadoa MJ, Martin Olivara C, Polo Dieza LM (2006) Indirect chiral HPLC determination and fluorimetric detection of D-amino acids in milk and oyster samples. J Dairy Sci 89(1): 82-89.

35. Erbe T, Bruckner H (2006) Chromatographic determination of amino acid enantiomers in beers and raw materials used for their manufacture. J Chromatogr A 881(1): 81-91. 
36. Kato S, Ishihara T, Hemmi H, Kobayashi H, Yoshimura T (2011) Alternations in D-amino acid concentrations and microbial community structures during the fermentation of red and white wines. J Biosci Bioeng 111(1): 104-108.

37. Mutaguchi Y, Ohmori T, Akano H, Doi K, Ohshima T (2003) Distribution of $\mathrm{D}$-amino acids in vinegars and involvement of lactic acid bacteria in the production of d-amino acids. Springerplus 2: 691.

38. Brückner H, Westhauser T (2003) Chromatographic determination of Land D-amino acids in plants. Amino Acids 24(1): 43-55.

39. Niedle A, Dunlop DS (1990) Developmental Changes in Free D-aspartic Acid in the Chicken Embryo and in the Neonatal Rat. Life Sci 46(21): 1517-1522.

40. Hashimoto A, Kumashiro S, Nishikawa, T, Oka T, Takahashi K, et al. (1993) Embryonic Development and Postnatal Changes in Free D-aspartate and D-serine in the Human Prefrontal Cortex. J Neuchem 61(1): 348-351.

41. Marchelli R, Dossena A, Palla G, Audhuy Peaudecerf M, Lefeuvre S, et al. (1992) D-Amino acids in reconstituted infant formula: A comparison between conventional and microwave heating. Science of Food and Agriculture 59(2): 217-226.

42. Sonnenburg JL, Bäckhed F (2016) Diet-microbiota interactions as moderators of human metabolism. Nature 535(7610): 56-64.

43. Bifari F, Ruocco C, Decimo I, Fumagalli G, Valerio A, et al. (2017) Amino acid supplements and metabolic health: a potential interplay between intestinal microbiota and systems control. Genes Nutr 12: 27.

44. Ballard A, Ahmad HO, Narduolo S, Rosa L, Chand N, et al. (2017) Quantitative Prediction of Rate Constants for Aqueous Racemization to Avoid Pointless Stereoselective Syntheses. Angew Chem Int Ed Engl 57(4): 982-985.

45. Pencharz PB, Ball RO (2004) Amino Acid Needs for Early Growth and Development. The Journal of Nutrition 134(6): 1566-1568.

46. Dallas DC, German JB (2017) Enzymes in human milk. Nestle Nutr Inst Workshop Ser 88: 129-136.

47. Khaldi N, Vijayakumar V, Dallas DC, Guerrero A, Wickramasinghe S, et al. (2014) Predicting the Important Enzymes in Human Breast Milk Digestion. J Agric Food Chem 62(29): 7225-7232.
48. Nielsen SD, Beverly RL, and Dallas DC. (2017) Milk Proteins are Predigested within the Human Mammary Gland. J Mammary Gland Biol Neoplasia 22(4): 251-261.

49. Brückner H, Jaek P, Langer M, Godel H (1992) Liquid chromatographic determination of D-amino acids in cheese and cow milk. Implication of starter cultures, amino acid racemases, and rumen microorganisms on formation, and nutritional considerations. Amino Acids 2(3): 271-284.

50. Hirabayashi Y, Furuya S (2008) Roles of L-serine and sphingolipid synthesis in brain development and neuronal survival. Prog Lipid Res 47(3): 188-203.

51. Esaki K, Sayano T, Sonoda C, Akagi T, Suzuki T, et al. (2015) L-Serine Deficiency Elicits Intracellular Accumulation of Cytotoxic Deoxysphingolipids and Lipid Body Formation. J Biol Chem 290(23): 14595-14609.

52. Moran LA (2008) Amino Acids and the Racemization "Problem"

53. Sasabe J, Suzuki M (2019) Distinctive Roles of D-Amino Acids in the Homochiral World: Chirality of Amino Acids Modulates Mammalian Physiology and Pathology. The Keio Journal of Medicine 10(3): 360.

54. Lonnie M, Hooker E, Brunstrom JM, Corfe BM, Green MA, et al. (2018) Protein for Life: Review of Optimal Protein Intake, Sustainable Dietary Sources and the Effect on Appetite in Ageing Adults. Nutrients 10(3): 360.

55. Lexell J, Taylor CC, Sjöström M (1988) What is the cause of the ageing atrophy? Total number, size and proportion of different fiber types studied in whole vastus lateralis muscle from 15- to 83-year-old men. J Neurol Sci 84(2):275-294.

56. Kimura T, Hesaka A, Yoshitaka Isaka Y (2020) D-Amino acids and kidney diseases. Clin Exp Nephrol 24(5): 404-410.

57. Sun, Miao S, Kai M, Jie W, Guangxian W et al. (2020) A Review of the Brain-Gut-Microbiome Axis and the Potential Role of Microbiota in Alzheimer's Disease. Journal of Alzheimer's Disease 73: (3): 849-865.

58. Chang CH, Lin CH, Hsien-Yuan Lane HY (2020) D-glutamate and Gut Microbiota in Alzheimer's Disease. Int J Mol Sci 21(8): 2676

59. Dyakin VV, Wisniewski TM, Lajtha A (2020) Chiral Interface of Amyloid Beta $(\mathrm{A} \beta)$ : Relevance to Protein Aging, Aggregation and Neurodegeneration. Symmetry 12(4): 585. 